\title{
Neural networks in art, sound and design
}

\author{
Juan Romero ${ }^{1}$ - Penousal Machado² \\ Published online: 26 October 2020 \\ (C) Springer-Verlag London Ltd., part of Springer Nature 2020
}

Artistic behavior and its manifestations are present in all human cultures that existed. The ubiquitous nature of art makes it a defining characteristic of the human species and intellect. Since the early days of Computing and Artificial Intelligence, pioneers such as Ada Byron or Alan Turing pondered about the possibility of creating machines able to perform artistic tasks and display creative behavior.

In recent years, the growth of the scientific community devoted to computational creativity and the developments in the field of machine learning - along with the increasing computational power-gave rise to several novel possibilities for the application of artificial neural networks (ANNs) to fields such as music, art, sound, architecture and design. In fact, although applications and techniques such as deep dreams, generative adversarial neural networks, style transfer and deep fakes are currently widespread, these were unattainable a few years ago.

The aim of this special issue is to put together a comprehensive group of papers that: represents the current trends on the field; reflects upon it; and identifies the core challenges and opportunities that lay ahead. With this in mind, the special issue includes three survey papers on the application of ANNs to music, games content and visual arts, respectively.

Jean-Pierre Briot, co-author of the book "Deep Learning Techniques for Music Generation," describes, in a pedagogical way, several innovative proposals for the generation of music through ANNs and deep learning. Briot takes us on

Juan Romero

juan.romero1@udc.es

Penousal Machado

machado@dei.uc.pt2

1 CITIC, Department of Computer Science, Faculty of Computer Science, University of A Coruña, 15071 A Coruña, Spain

2 Department of Informatics Engineering, Centre for Informatics and Systems of the University of Coimbra, University of Coimbra, Coimbra, Portugal a journey from the first works of the late 80s that used ANNs for the generation of music to recent works that illustrate the variety of applications and techniques that arise today.

Jialin Liu et al. analyze the deep learning revolution through two converging trends in the last years: the increasing use of deep learning techniques-such as generative adversarial network, variational autoencoders and recurrent neural networks-for generative tasks in nongame-related contexts; the increasing use of deep learning in the procedural content generation. They focus on the latter trend to point out relevant research issues and deep learning architectures used for the generation of different types of content.

Santos et al. present a state of the art that extensively explores the use of ANNS and deep learning techniques in visual art. The works are divided in several categories: works that detect objects in artistic images; those that classify based on style or author; research that classifies or predicts aesthetic quality or value; and systems that generate images. In total, 92 articles are cited from the years 2012-2020, presenting a thorough overview of this area.

Two other articles present innovative research on the use of deep learning for individual aesthetics and in audio synthesis for music composition and sound design applications, respectively.

Jon McCormack and Andy Lomas seek to progress machine-assisted aesthetic judgment based on an artist's personal aesthetic preferences. To do so, they study how recent advances in deep learning can help automate personal aesthetic judgment in order to avoid the problems associated with the interactive genetic algorithm.

Finally, Tatar et al. present Latent Timbre Synthesis, an open source application based on a new method of audio synthesis that uses deep learning.

This application allows professionals to generate timbres between two audio extracts of their selection using interpolation and extrapolation in the latent space of the audio frames.

Publisher's Note Springer Nature remains neutral with regard to jurisdictional claims in published maps and institutional affiliations. 